\title{
Haptoglobin Decreased
}

National Cancer Institute

\section{Source}

National Cancer Institute. Haptoglobin Decreased. NCI Thesaurus. Code C78330.

A laboratory test result which indicates decreased levels of haptog lobin in a biological specimen. 